\title{
Early-phase trials of lipoprotein(a) lowering
}

In phase I-II clinical trials, antisense oligonucleotides targeting apolipoprotein(a) mRNA have produced large reductions in the level of lipoprotein(a) - a major risk factor for cardiovascular disease and calcific aortic valve stenosis. Lipoprotein(a) is a modified LDL particle composed of LDL and apolipoprotein(a).

No approved therapy for substantially lowering lipoprotein(a) concentrations currently exists. Two antisense oligonucleotides from Ionis Pharmaceuticals have now been produced to fill this unmet clinical need.

IONIS-APO $(a)_{\mathrm{Rx}}$ has previously undergone phase I clinical testing. In the phase II trial now reported in The Lancet, 64 patients with elevated lipoprotein(a) levels (125-437 nmol/l in cohort $A ; \geq 438 \mathrm{nmol} / \mathrm{l}$ in cohort $\mathrm{B}$ ) were randomly assigned to an escalating dose (100-30 subcutaneous IONIS-A injection of subcutaneol once per week for 12 we 85-99 days, patients rec IONIS-APO (a $)_{\text {Rx }}$ had a tion in lipoprotein(a) le cohort $\mathrm{A}$ and $71.6 \%$ in c $P<0.0001$ versus pooled .

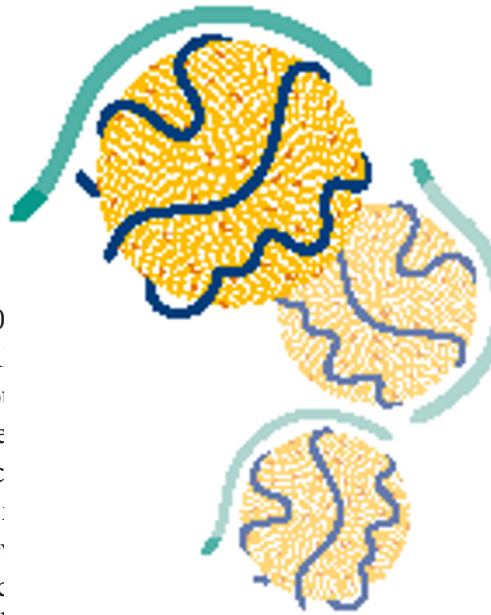

IONIS-APO(a)- $\mathrm{L}_{\mathrm{Rx}}$ is a ligand-conjugated antisense oligonucleotide, modified to be highly and selectively taken up by hepatocytes. In a first-in-human phase I/IIa trial, published in the same Lancet article, 58 healthy volunteers with a lipoprotein(a) level $\geq 75 \mathrm{nmol} / 1$ were randomly assigned to receive a single dose (10-120 mg) or multiple doses $(10 \mathrm{mg}, 20 \mathrm{mg}$, or $40 \mathrm{mg}$ ) of subcutaneous IONIS-APO(a)- $\mathrm{L}_{\mathrm{Rx}}$ in an ascending-dose design, or placebo.
At day 30 in individuals who received single doses of IONIS-APO(a)- $\mathrm{L}_{\mathrm{Rx}}$, significant dose-dependent reductions in mean lipoprotein(a) level occurred in all groups. Similarly, at day 36 in individuals who received multiple doses of IONIS-APO(a)- $\mathrm{L}_{\mathrm{Rx}}$, the mean lipoprotein(a) level was reduced by $66 \%$ in the $10 \mathrm{mg}$ group, $80 \%$ in the $20 \mathrm{mg}$ group, and $92 \%$ in the $40 \mathrm{mg}$ group $(P=0.0007$ for each versus placebo). Both antisense oligonucleotides were safe.

These results are "a major advance in both the specific targeting of lipoprotein(a) and in tolerability," comments Sotirios Tsimikas, senior author on the publication of the trials. "Future trials are being planned to assess whether the drugs can reduce cardiovascular disease events and the progression of calcific aortic valve stenosis."

Gregory B. Lim

ORIGINAL ARTICLE Viney, N. J. et al. Antisens oligonucleotides targeting apolipoprotein(a) in people with raised lipoprotein(a): two randomised, double-blind, placebo-controlled, dose-ranging trials. Lancet http://dx.doi.org/10.1016/S01406736(16)31009-1 (2016) 